\title{
Dynamic Importance Analysis of Components with Known Failure Contribution of Complex Systems
}

\author{
Yangfan Li, Yingjie Zhang $\mathbb{D}^{D}$, Bochao Dai, and Lin Zhang \\ School of Mechanical Engineering, Xian Jiaotong University, Xian, China \\ Correspondence should be addressed to Yingjie Zhang; yjzhang@xjtu.edu.cn
}

Received 28 December 2017; Revised 1 March 2018; Accepted 20 March 2018; Published 24 April 2018

Academic Editor: Alessandro Lo Schiavo

Copyright (C) 2018 Yangfan Li et al. This is an open access article distributed under the Creative Commons Attribution License, which permits unrestricted use, distribution, and reproduction in any medium, provided the original work is properly cited.

\begin{abstract}
Importance analysis deals with the investigation of influence of individual system component on system operation. This paper mainly focuses on dynamic important analysis of components in a multistate system. Assuming that failure probabilities of system components are independent, a new time integral-based importance measure approach (TIIM) is proposed to measure the loss of system performance that is caused by each individual component. Reversely the importance of a component can be evaluated according to the magnitude of performance loss of the system caused by it. Moreover, the dynamic varying curve of importance of a component with time can be described by calculating criticality of the component at each state. On the other hand, in the proposed approach, the importance probability curve of a component is fitted by using the failure data from all components of system excluding that of the component itself so as to solve the problem of inaccurate fitting caused by small sample data. The approach has been verified by probability analysis of failure data of CNC machines.
\end{abstract}

\section{Introduction}

Importance measures have been widely used for identifying system weaknesses and supporting system improvement activities from the design viewpoint. With the importance values of all components, proper actions can be taken on the weakest component to improve system reliability at the minimum cost or effort [1].

Every component of a system has different contribution to the system performance. Investigation of this influence is the major objective of importance analysis, which can be qualitative or quantitative. From the viewpoint of classical reliability, a system is always regarded as binary-state. That is, all components only have two states of "working perfect" or "completely failed." This representation is suitable for systems where any deviation from perfect functioning may cause a disaster, for example, nuclear power plants [2] or aviation systems [3]. Barlow and Proschan [4] proposed an importance measure for the components in a coherent system and the basis events in a fault tree is defined. However, it is not appropriate for studying systems that work at several performance levels, for example, process investigation of gradually degrading systems. It is also not very suitable for the analysis of a complex system which has many heterogeneous factors such as hardware, software, organizational structure, and human factors [5].

Unlike binary-state systems that are either perfectly working or completely failed, newer approaches show that typical components of a system may work at many performance loss levels from zero to one, each level with a certain probability. Despite a system like CNC machine tools can be called a multistate system (MSS) that has a changing situation over time, many of the results for the binary case can be computed for multistate systems using the binary structure and reliability function concepts [6]. Researchers such as Lisnianski and Levitin $[7,8]$, Nourelfath et al. $[9,10]$, Murchland [11], and Hudson and Kapur [12] have studied it in depth.

Birnbaum [13] introduced two basic importance measures (IM), that is, structural importance (SI) and Birnbaum importance (BI), and one of the first generalizations of SI and BI has been proposed for MSS [14]. These papers introduced various definitions of several basic IM [14-17]. Each of the definitions has a specific physical meaning; for example, one type of these measures allows finding component state by its 
influence degree on the whole system [15], but another one allows quantifying importance of individual states of a given component on a specific system state [14, 16], while there are still some methods that permit investigating the total importance of a given component [17]. In most cases, UGF (universal generating function) approach can be used for importance analyses of high dimensional systems. However, in most cases, the system structural details are unknown, which makes it difficult to use the UGF method because this method is based on a moment generating function that is mathematically defined and depends on the working context and the system structure. It implies that these computing methods add specifics to the original definitions of IM.

In recent years, many researchers have made contributions to multistate system IM analysis. They focused on developing different approaches to permit setting limitations of the calculation methods. For example, the IM for complex systems with multiple components was proposed in [18]; Cadini et al. [19] developed Monte-Carlo method for the analysis of systems with small failure probabilities. Zhang et al. [20] proposed an approach to measure component importance of manufacturing systems based on failure losses. As an application example, a piston production line was chosen for evaluation purpose [21]. Dui et al. [22] extend the integrated importance measure (IIM) from unit time to system lifetime and to different life stages. Levitin et al. [23] proposed an approach based on performance level restrictions to evaluate the commonly used importance measures. Kvassay et al. [24] developed new approach which can be used for calculation of all types of critical states and it is based on the application of direct partial logic derivatives. In addition, many researchers focus on the importance measures of $k$-out-of- $n$ system [25, 26]. Moreover, the most comprehensive research of current work in importance analysis was conducted in $[27,28]$.

This paper aims to seek an efficient method for evaluating component importance of complex system as a multistate system. With the loss of system performance of complex system, one of the open questions is how to accurately calculate the dynamic importance of a component. Traditional importance measures mainly concern the change of system performance caused by the reliability change of components, but they seldom consider the joint effect of probability distributions, transition intensities of the object component states, and the loss of system performance. As a matter of fact, the expected loss of system performance from failures is related to the expected number of component failures and the effect of system structure. It means that some new mathematical approaches for known IM need to be studied. In this paper, considering how the transition of component states affects the system' mean time to failure (MTTF), we study the time integral importance measure (TIIM) to evaluate the importance of components of system.

The remainder of this paper is organized as follows. The traditional importance measures are analyzed in Section 2. The TIIM of component states is described in Section 3. Section 4 demonstrates the application of TIIM on CNC machine tools. The conclusions and future work are given in Section 5 .

\section{Traditional Importance Measures}

Comparison of traditional methods is shown in Table 1; although several papers [24, 27] have reviewed traditional importance measures, it is seen that these papers cover various categories with different emphases. In this paper, the dynamic characteristics of the traditional methods are compared.

From Table $1, I^{\mathrm{B}}(i)$ will change along with the component working; the physical meaning of $I^{\mathrm{B}}(i)$ is the sensitivity of the system due to deterioration of component $i$ in unit time. the Barlow-Proschan measure is a weighted average of the Birnbaum measure, with the weight at time $t$ being $f_{t}(i)$. According to this measure, the more a component is important, the more likely it is the direct cause of system failure. $I_{s}^{\mathrm{G}}(i)$ can be interpreted as the change of the system performance when a component deteriorates from state $s$ to $s-1$. $I_{s}^{\mathrm{W}}(i)$ represents the contribution of state $s$ of component $i$ to the system performance. Natvig importance measure [29], like the Barlow-Proschan measure, is a weighted average of the Birnbaum measure [30] and is static with the mean lifetime and repair time [1]. $I_{s, 0}^{\mathrm{IIM}}(i)$ can be interpreted as the rate of system performance reduction due to deterioration of component $i$ from state $s$ to state 0 .

\section{Modeling of Time Integral Importance Measure (TIIM)}

Some assumptions are described as follows:

(1) $x_{i}, i=1,2, \ldots, M$, are components of system.

(2) All components and a system under consideration have the set of reliability states $\{0,1, \ldots, S\}, S \geq 1$.

(3) The reliability states are ordered: the state 0 is the worst and the state $S$ is the best.

(4) The component and the system reliability states degrade with time $t$.

(5) $E_{i}(s), i=1,2, \ldots, M$, are independent random variables representing the lifetime of components $x_{i}$ in the reliability state subset $\{s, s+1, \ldots, S\}$, while they were in the reliability state $S$ at the moment $t=0$.

(6) $E(s)$ is a random variable representing the lifetime of a system in the reliability state subset $\{s, s+1, \ldots, S\}$, while it was in the reliability state $S$ at the moment $t=0$.

(7) $s_{i}(t)$ is a component $x_{i}$ reliability state at the moment $t, t=[0, \infty)$, given that it was in the reliability state $S$ at the moment $t=0$.

(8) $s(t)$ is the system reliability state at the moment $t, t=$ $[0, \infty)$, given that it was in the reliability state $S$ at the moment $t=0$.

(9) The above assumptions mean that the reliability states of the ageing system and component may change over time only from better to worse. 


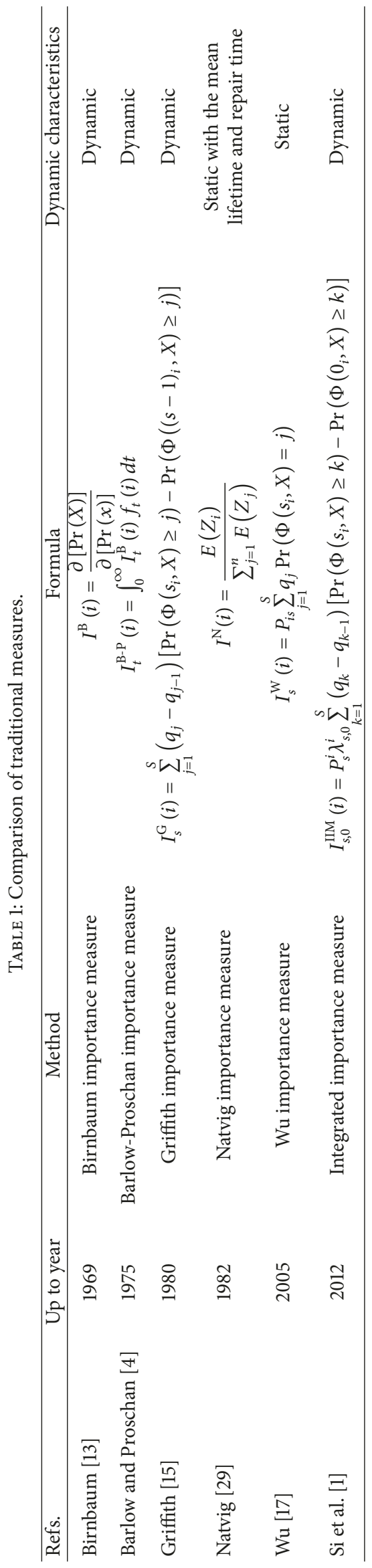


Definition 1. A vector

$$
\begin{aligned}
& R_{i}(t, \cdot)=\left[R_{i}(t, 0), R_{i}(t, 1), \ldots, R_{i}(t, S)\right], \\
& \qquad \text { for } t \in[0, \infty), i=1,2, \ldots, M,
\end{aligned}
$$

where

$$
R_{i}(t, s)=P\left(s_{i}(t) \geq s \mid s_{i}(0)=S\right)=P\left(T_{i}(s)>t\right)
$$

for $t \in[0, \infty) ; s=0,1, \ldots, S$ is the reliability that the component $x_{i}$ is in the state subset $\{s, s+1, \ldots, S\}$ at the moment $t, t \in[0, \infty)$, while being in the state $S$ at the moment $t=0$ is called the multistate reliability function of a component $x_{i}$.

Definition 2. A vector

$$
R(t, \cdot)=[R(t, 0), R(t, 1), \ldots, R(t, S)], \quad t \in[0, \infty),
$$

where

$$
R(t, s)=P(s(t) \geq s \mid s(0)=S)=P(T(s)>t)
$$

for $t \in[0, \infty) ; s=0,1, \ldots, S$ is the reliability that the system is in the state subset $\{s, s+1, \ldots, S\}$ at the moment $t, t \in[0, \infty)$, while being in the reliability state $S$ at the moment $t=0$ is called the multistate reliability function of a system.

It is clear that from Definitions 1 and 2 , for $s=0$, we have $R_{i}(t, 0)=1$ and $R(t, 0)=1$.

Under the above definitions, the mean time to failure (MTTF) of the system $E(s)$ in the reliability state subset $\{s, s+$ $1, \ldots, S\}$ is given by

$$
E(s)=\int_{0}^{\infty} R(t, s) d t, \quad s=1,2, \ldots, S .
$$

Moreover, the mean time to failure (MTTF) of the component $i E_{i}(s)$ in the reliability state subset $\{s, s+1, \ldots, S\}$ is given by

$$
E_{i}(s)=\int_{0}^{\infty} R_{i}(t, s) d t, \quad s=1,2, \ldots, S .
$$

Let $x_{i}(k)\left(i=1,2, \ldots, M ; k \in N^{+}\right)$represent the $k$ th kind failure of component $i$. Assume that all failure data conform to the function $\psi[k]$; then the reliability of system is

$$
R(t, \cdot)=\psi\left(\sum_{i=1}^{M} \sum_{k=1}^{N} x_{i}(k)\right), \quad t \in[0, \infty) .
$$

In order to avoid the inaccurate evaluation caused by the few failure data, we wiped off the failure data of component $i$; it means component $i$ is working well. The obtained evaluation result is compared with original failure data so that we can evaluate the influence of component $i$ on the system. If we wiped off the failure data of component $i$, the reliability of system would be

$$
R_{i}(t, \cdot)=\psi_{i}\left(\sum_{j=1}^{M} \sum_{k=1}^{N} x_{j}(k)-\sum_{k=1}^{N} x_{i}(k)\right), \quad i \neq j .
$$

Assume that $U_{i}(s)$ represents the rate of system performance loss due to deterioration of the component $i$ from state $s$ to state $0 . N_{i}(s)$ represents the expected times at which the component $i$ falls from state $s$ to state 0 in unit time. $C_{i}(s)$ represents the expected change of the system performance when component falls from state $s$ to state 0 based on the probability of state $s$ of component $i$. We get

$$
U_{i}(s)=N_{i}(s) \times C_{i}(s) .
$$

Zhang et al. [31] give the following equation:

$$
\operatorname{Pr}\{t \leq T \leq t+\Delta t\}=R(t)-R(t+\Delta t) .
$$

The integral difference in reliabilities between the system and a component is measured in this paper, so we get

$$
C_{i}(s)=\left|E_{i}(s)-E(s)\right|=\int_{0}^{t}\left|R_{i}(t)-R(t)\right| d t .
$$

And $N_{i}(s)=\lambda_{s}^{i}$, in which $\lambda_{s}^{i}$ represents the expected times at which component $i$ falls at state $s$ in unit time, so we have

$$
\begin{aligned}
U_{i}(s) & =N_{i}(s) \times C_{i}(s)=\lambda_{s}^{i} \int_{t}^{t+\Delta t}\left|R_{i}(t)-R(t)\right| d t, \\
I_{i}^{\mathrm{TIIM}}(s) & =\frac{\int_{t}^{t+\Delta t}\left|R_{i}(t)-R(t)\right| d t}{1 / \lambda_{s}^{i}}=U_{i}(s) .
\end{aligned}
$$

From (13), $I_{i}^{\mathrm{TIIM}}(s)$ represents the rate of the loss of system performance due to deterioration of component $i$ at state $s$. Therefore, if the state $s$ of component $i$ has the minimal value of $I_{i}^{\mathrm{TIIM}}(s)$, it means the smallest loss of system performance due to deterioration of component $i$ at state $s$.

By normalizing the parameters, $I_{i}^{\mathrm{TIIM}}(s)$ is calculated, which can formulated as

$$
I_{i}^{\mathrm{TIIM}}(s)=\frac{I_{i}^{\mathrm{TIIM}}(s)+I_{i \max }^{\mathrm{TIIM}}(s)}{I_{i \min x}^{\mathrm{TIIM}}(s)+I_{i \max }^{\mathrm{TIIM}}(s)},
$$

where $I_{i \min }^{\mathrm{TIIM}}(s)$ represents the minimum value in all components and $I_{i \max }^{\mathrm{TIIM}}(s)$ represents the maximum value in all components; $I_{i \min }^{\mathrm{TIIM}}(s)=\min _{0<i<M} I_{i}^{\mathrm{TIIM}}(s), I_{i \max }^{\mathrm{TIIM}}(s)=$ $\max _{0<i<M} I_{i}^{\mathrm{TIIM}}(s)$.

Thus, the importance of the component $i$ is evaluated by (13) and (14).

\section{Verification by Examples}

This paper collected the failure data of CNC machine tools for fifteen months. Table 2 shows the goodness-of-fit of the failure data in Minitab software. It has been clearly seen that the data can be better fitted as a three-parameter Weibull distribution.

As evaluated by Minitab, the mean time to failure (MTTF) of CNC machine tools is $876.8 \mathrm{~h}$, as shown in Figure 1. According to three-parameter Weibull distribution, the shape parameter $\beta=0.872251$, the scale parameter $\theta=686.185$, 
TABLE 2: Goodness-of-fit.

\begin{tabular}{lcc}
\hline Distribution & Anderson-Darling & Rank \\
\hline Weibull & 2.551 & 4 \\
Lognormal & 2.327 & 2 \\
Exponential & 3.450 & 8 \\
Log Logistic & 2.488 & 3 \\
3-Parameter Weibull & 1.216 & 1 \\
3-Parameter Lognormal & 2.667 & 6 \\
2-Parameter Exponential & 2.839 & 7 \\
3-Parameter Log Logistic & 2.591 & 5 \\
Smallest extreme value & 12.776 & 11 \\
Normal & 7.959 & 10 \\
Logistic & 6.507 & 9 \\
\hline
\end{tabular}

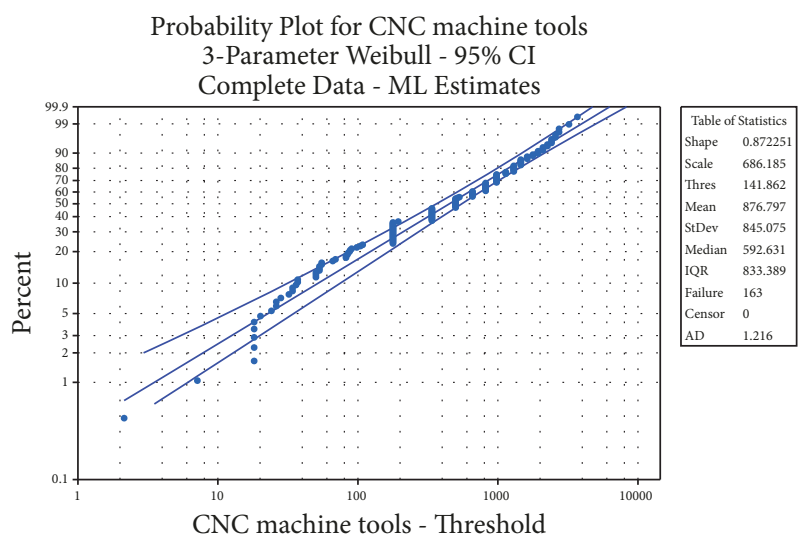

FIgUre 1: Probability plot of MTTF of CNC machine tools.

and the threshold $t_{0}=141.862$, so the equations of system were gotten as follows:

$$
\begin{aligned}
& R(t)=\exp \left[-\left(\frac{t-t_{0}}{\theta}\right)^{\beta}\right], \quad t \geq t_{0}, \\
& \lambda(t)=\frac{\beta}{\theta}\left(\frac{t-t_{0}}{\theta}\right)^{\beta-1}, \quad t \geq t_{0} .
\end{aligned}
$$

Figure 2 shows the probability plots for MTTF of CNC control system, turning tool carry, power supply and electrical system, chip-removal system, lubrication system, hydraulic system, main transmission system, and clamp system. For convenience of calculations, we use the MTTF of CNC machine tools without any component as the system performance changes due to the component.

According to Figure 2, the lifetime and repair time of components are consistent with three-parameter Weibull distribution. The time period of $t$ is set to 350 hours. Going by the time period the machine will reach steady state. The failure rate is the inverses of the mean lifetimes $\left(\lambda_{1,0}^{i}=\right.$ $\left.1 / \mathrm{ML}_{i}\right)$, while the repair rate is the inverses of the mean repair times $\left(\mu_{1,0}^{i}=1 / \mathrm{MR}_{i}\right)$.
When a machine operates into steady state we can get the equations

$$
\begin{aligned}
& 0=P_{i 1} \lambda_{1,0}^{i}-P_{i 0} \mu_{0,1}^{i} \\
& 1=P_{i, 1}+P_{i, 0}
\end{aligned}
$$

and the obtained component importance results are listed in Table 3.

From Table 3, the component power supply and electrical system have the largest importance in Birnbaum importance measures, Barlow-Proschan importance measures, and Integrated importance measure because the power supply or electrical system is the least reliable unit in a series system. The reason is that the Birnbaum importance does not consider the mean lifetime or criticality. In addition, integrated importance measure is generalizations of Birnbaum importance measure; it is not good to solve the component importance of small sample.

Although power supply or electrical system is the least reliable unit, tool carry has a smaller mean time to failure (MTTF) or a larger criticality than power supply and electrical system. Therefore, from the TIIM, Natvig importance measure, and $\mathrm{Wu}$ importance measure, the importance of tool carry is larger than that of the power supply and electrical system.

Because of the performance loss of CNC machine tools, the importance of components may be changing. Therefore, the case of $t=300$ hours may be difficult to justify empirically. Here, we discuss the case of $t=1300 \mathrm{~h}$.

For $t=1300 \mathrm{~h}$, assuming that the system is a nonrepairable system, all the parameters are required in the simulations; as shown in Table 4, both the ranks of time integral importance and the Natvig importance are all the same and tool carry has the largest time integral importance. Comparing with Table 3 , the rank of $\mathrm{CNC}$ control system changes from 8 to 6 , and the hydraulic system becomes the smallest importance component.

As shown in Tables 3 and 4, Natvig importance is the same, which proves that the Natvig importance is static with the mean lifetime and repair time. Birnbaum importance measure, integrated importance measure, and TIIM are sensitive to working hours, but the Natvig importance measure is the mean importance of a component's whole life cycle. The values of the Wu importance vary with time while the rank of the components is constant. The reason is that $I_{s}^{\mathrm{Wu}}(i)$ represents the contribution of component $i$ in the system from state $s$ to state 0 . Wu importance is a static indicator of the system performance [1]. The rank of Barlow-Proschan importance is constant. This is because Barlow-Proschan importance measure has a stationary probability, or the failure of the component can always cause the failure of the system. It shows that Barlow-Proschan importance measure is a static indicator of system failure, so it is suggested that researchers select the proper one for different improvement actions.

In the case the reliability loss of a CNC machine tool with time is shown in Figure 3. As shown in Figure 4, the time integral importance of components is changed along with the loss of system performance, where the steady state ranges 


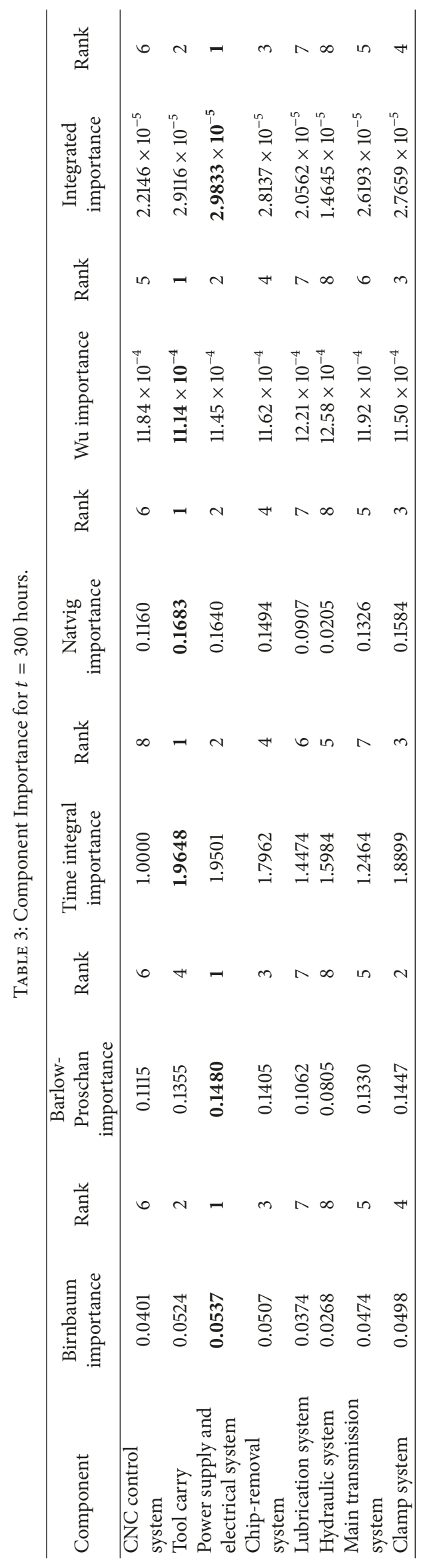




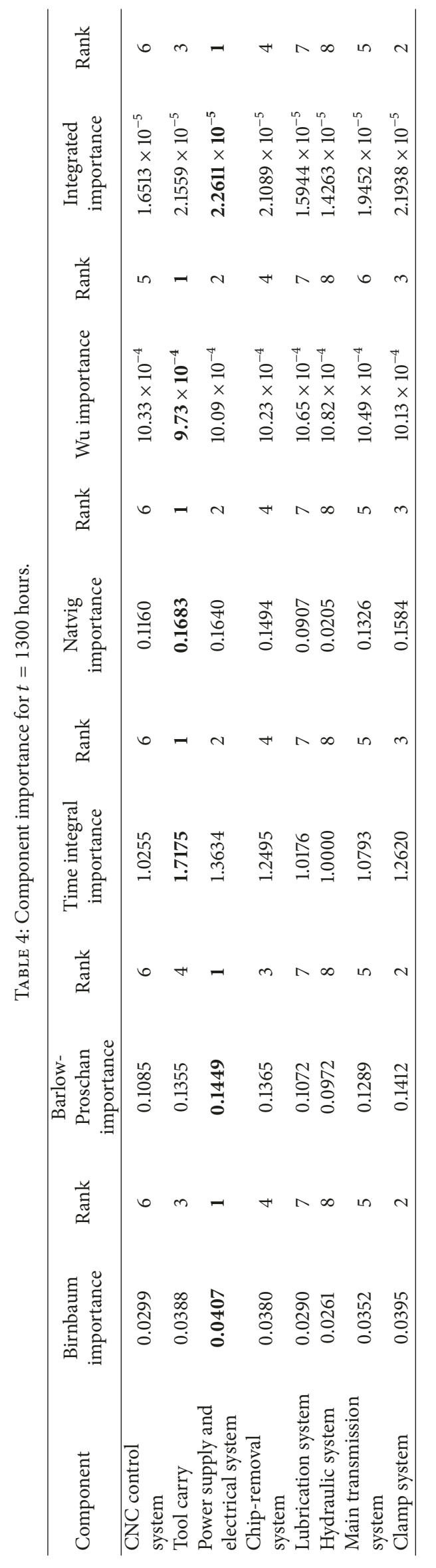




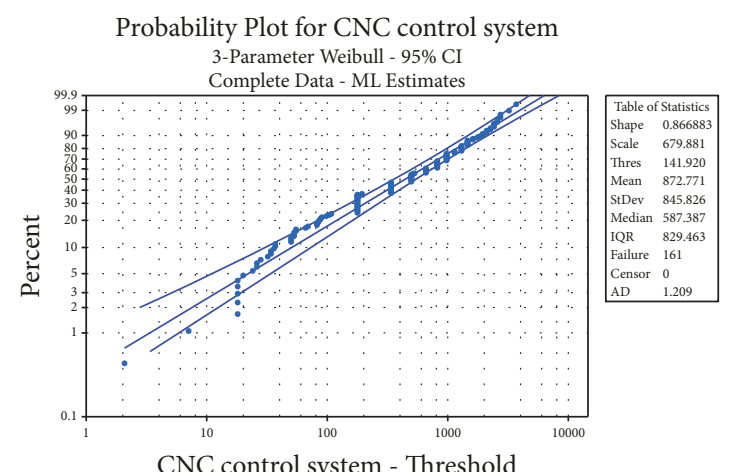

(a) Probability plot of MTTF of CNC machine tools with removal of CNC control system

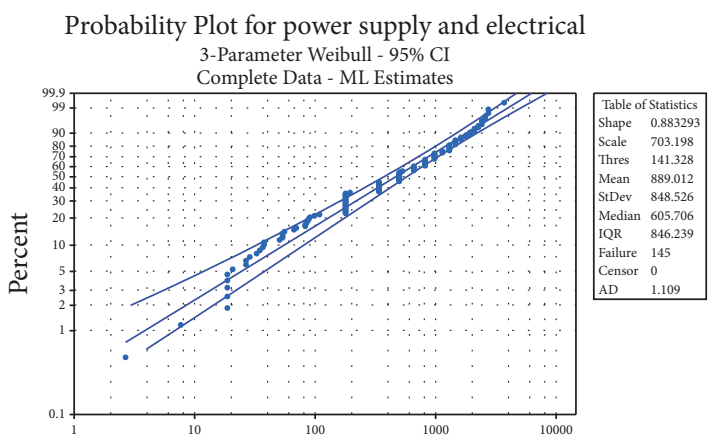

power supply and electrical - Threshold

(c) Probability plot of MTTF of CNC machine tools with removal of power supply and electrical system
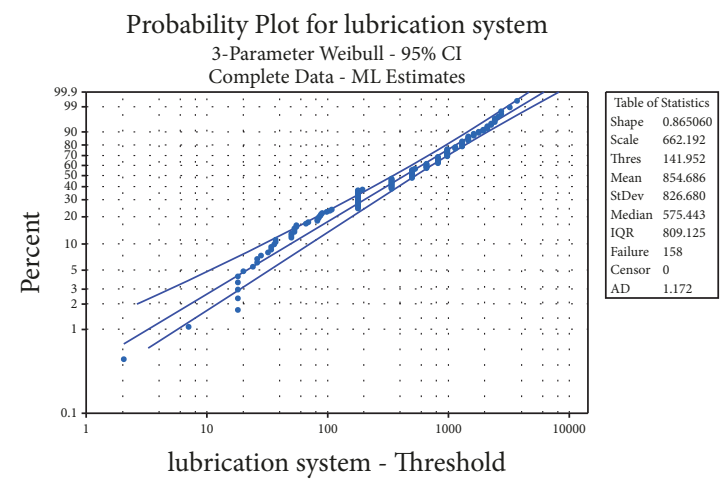

(e) Probability plot of MTTF of CNC machine tools with removal of lubrication system

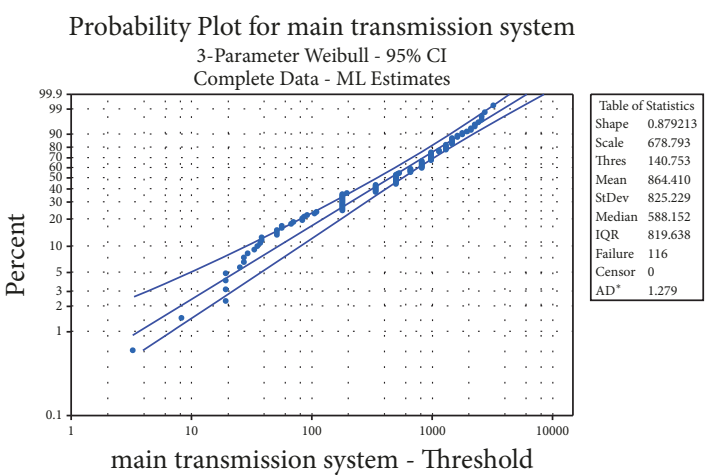

(g) Probability plot of MTTF of CNC machine tools with removal of main transmission system

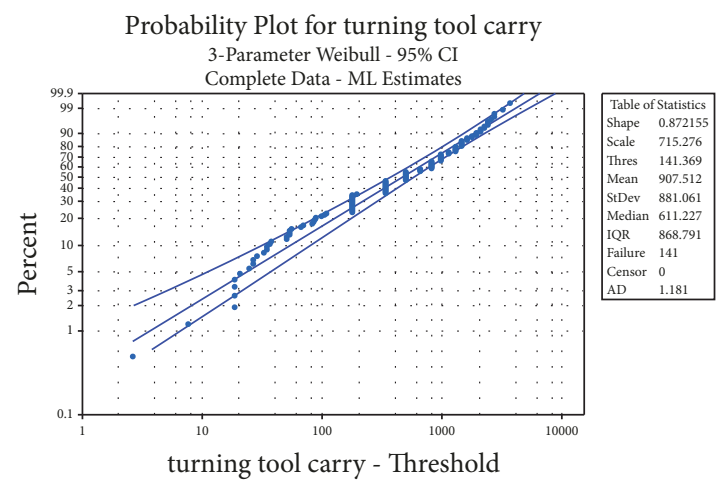

(b) Probability plot of MTTF of CNC machine tools with removal of turning tool carry

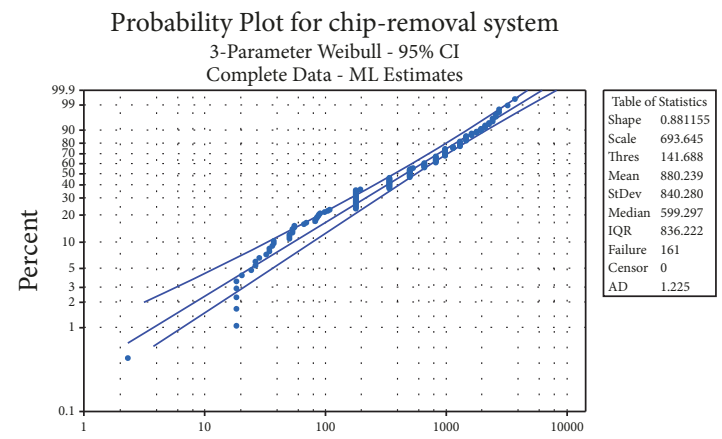

chip-removal system - Threshold

(d) Probability plot of MTTF of CNC machine tools with removal of chip-removal system

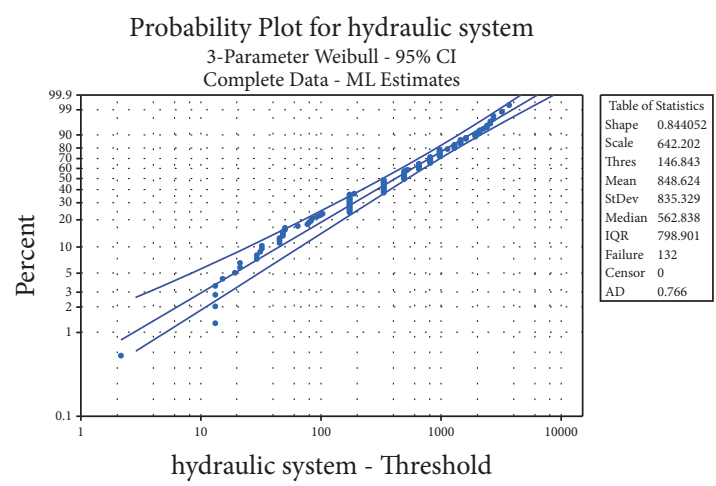

(f) Probability plot of MTTF of CNC machine tools with removal of hydraulic system

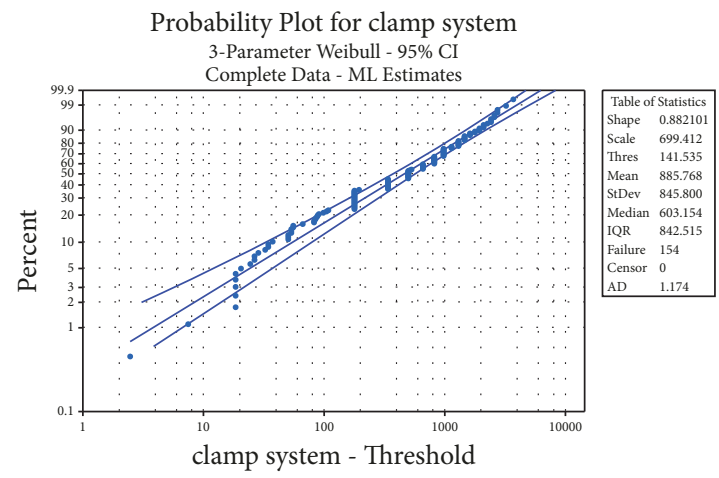

(h) Probability plot of MTTF of CNC machine tools with removal of clamp system

FIGURE 2: Probability plot of MTTF of components. 


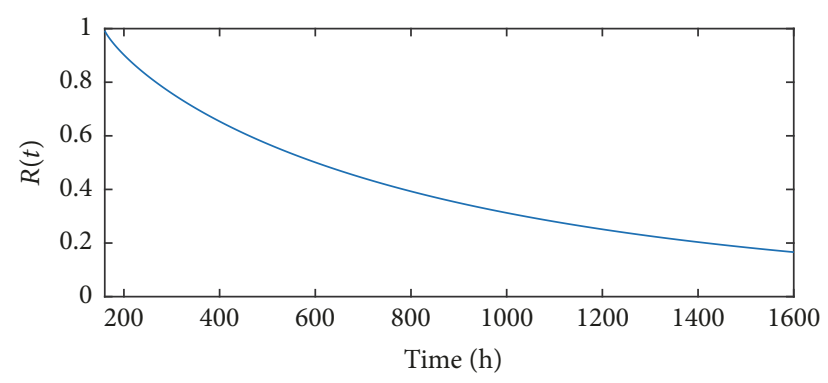

FIGURE 3: Reliability change of CNC machine tools.

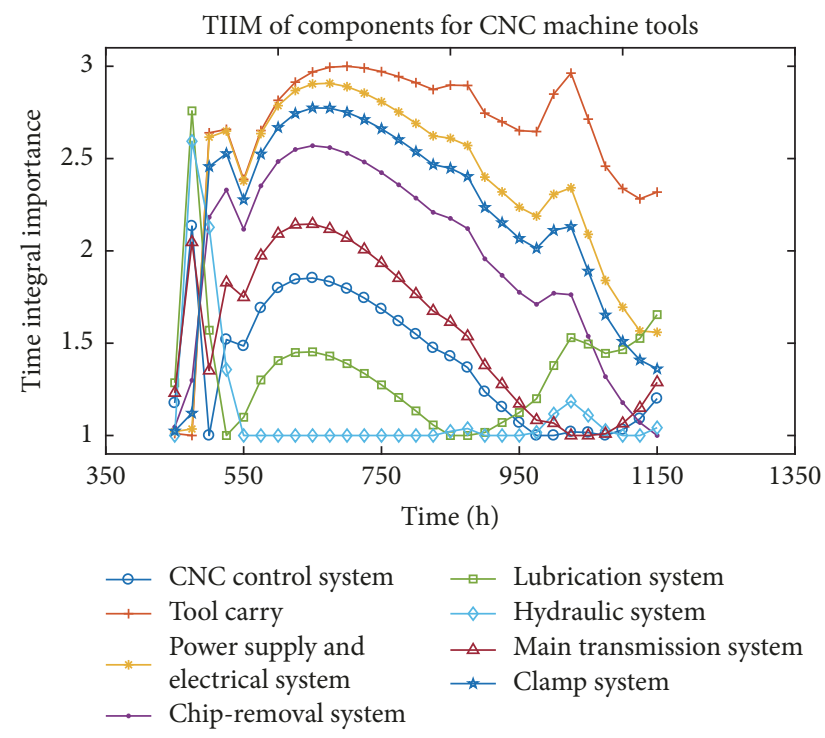

FIGURE 4: TIIM change of components for CNC machine tools.

from $t=350 \mathrm{~h}$ to $t=1350 \mathrm{~h}$. In this state, the ranking of TIIM of components is almost the same. The ranking of TIIM can help designers and managers to identify the most important component of the system. In particular, it can help them to find out the most responsible component for the performance loss of system at arbitrary state.

Known from the definition of TIIM, the proposed method can be used for importance evaluations of major components of a machine in binary and multiple states, respectively. Therefore, the conclusions derived from multistate systems can also be used for binary-state ones.

\section{Conclusions and Future Works}

This paper has discussed the TIIM of component states based on the loss of system performance. First, we present the definition of time integral importance measure (TIIM) of component states. Then the proposed method is compared with the existing approaches, such as Birnbaum importance, $\mathrm{Wu}$ importance, and Natvig importance. Finally, the proposed method is verified by a type of CNC machine tools. The major conclusions obtained are summarized as follows:

(1) To evaluate the influence of a component of complex mechanical system on system' mean time to failure (MTTF), the component reliability fits with the failure data which is removal of the component fault data in the whole failure data; in this way, TIIM can avoid the problem of inaccurate fitting due to small sample data. Then, the integral difference in reliabilities between the system and a component is measured for the evaluation purpose. In comparison with the existing method, the coupling relations among components is not required and the computational complexity is reduced greatly.

(2) TIIM is a new dynamic importance measure. In comparison with traditional importance measures, criticality of a component is taken into account by the computation of the component failure probability, and the calculation results are more practical.

(3) Although a component at a specific state has great influence on system performance, the influence may be little at the other states. Therefore, TIIM can evaluate the component importance in its whole lifetime and find out the most responsible component for system performance loss. Then, the component could be monitored to improve system performance.

In addition, the proposed approach in this study is only the first step of the importance measure of components of complex system. Future researches are needed to confirm the component states $(s, s-1, \ldots, 0)$ based on system performance changes.

\section{Notations}

CNC: Computer numerical control

MTTF: Mean time to failure

MSS: Multistate systems

IM: Importance measure

SI: $\quad$ Structural importance

BI: $\quad$ Birnbaum importance

UGF: Universal generating function

TIIM: Time integral importance measure

$M: \quad$ Number of components in a system

$i$ : $\quad$ Index of component $i, i=1,2, \ldots, M$

$q_{s}: \quad$ The performance level corresponding to

state $s$ of a system

E: $\quad$ The expectation

$U: \quad$ The expected performance of a system

$X: \quad\left(x_{1}, x_{2}, \ldots, x_{n}\right)$ : state vector of the components

$P_{i s}: \quad \operatorname{Pr}\left\{x_{i}=s\right\}, s=0,1,2, \ldots, S_{i}$

$R_{i}(t)$ : The reliability of component $i$ at lifetime $t$

$\mathrm{ML}_{i}$ : The mean lifetime of component $i$

$\mathrm{MR}_{i}$ : The mean repair time of component $i$

$I^{\mathrm{B}}(i)$ : Birnbaum importance of component $i$

$I^{\mathrm{G}}(i)$ : Griffith importance of component $i$

$I^{\mathrm{Wu}}(i)$ : Wu importance of component $i$

$I^{\mathrm{N}}(i)$ : Natvig importance of component $i$ $I^{\text {TIIM }}(i)$ : Time integral importance of component $i$.

\section{Conflicts of Interest}

The authors declare that they have no conflicts of interest. 


\section{Acknowledgments}

This work is supported by the Natural Science Basic Research Plan in Shaanxi Province of China (Grant no. 2017ZDJC-21) and Open Project for Chinese Institute for Quality Research, SJTU (Grant no. 2016-05).

\section{References}

[1] S. Si, H. Dui, X. Zhao, S. Zhang, and S. Sun, "Integrated importance measure of component states based on loss of system performance," IEEE Transactions on Reliability, vol. 61, no. 1, pp. 192-202, 2012.

[2] Y. Watanabe, T. Oikawa, and K. Muramatsu, "Development of the DQFM method to consider the effect of correlation of component failures in seismic PSA of nuclear power plant," Reliability Engineering \& System Safety, vol. 79, no. 3, pp. 265-279, 2003.

[3] B. Nyström, L. Austrin, N. Ankarbäck, and E. Nilsson, "Fault tree analysis of an aircraft electric power supply system to electrical actuators," in Proceedings of the 2006 9th International Conference on Probabilistic Methods Applied to Power Systems, PMAPS, Stockholm, Sweden, June 2006.

[4] R. E. Barlow and F. Proschan, "Importance of system components and fault tree events," Stochastic Processes and Their Applications, vol. 3, pp. 153-173, 1975.

[5] E. Zio, "Reliability engineering: old problems and new challenges," Reliability Engineering \& System Safety, vol. 94, no. 2, pp. 125-141, 2009.

[6] R. E. Barlow and A. S. Wu, "Coherent systems with multistate components," Mathematics of Operations Research, vol. 3, no. 4, pp. 275-281, 1978.

[7] A. Lisnianski, "Application of extended universal generating function technique to dynamic reliability analysis of a multistate system," in Proceedings of the 2nd IEEE International Symposium on Stochastic Models in Reliability Engineering, Life Science and Operations Management (SMRLO '16), pp. 1-10, Beer Sheva, Israel, February 2016.

[8] A. Lisnianski and G. Levitin, Multi-State System Reliability: Assessment, Optimization and Applications, vol. 6 of Series on Quality, Reliability and Engineering Statistics, World Scientific Publishers, Singapore, 2003.

[9] M. Nourelfath and D. Ait-Kadi, "Optimization of series-parallel multi-state systems under maintenance policies," Reliability Engineering \& System Safety, vol. 92, no. 12, pp. 1620-1626, 2007.

[10] M. Nourelfath and Y. Dutuit, "A combined approach to solve the redundancy optimization problem for multi-state systems under repair policies," Reliability Engineering \& System Safety, vol. 86, no. 3, pp. 205-213, 2004.

[11] J. D. Murchland, "Fundamental concepts and relations for reliability analysis of multi-state systems," Reliability and fault tree analysis (Conf., Univ. CALifornia, Berkeley, CALif., 1974), Soc. Indust. Appl. Math., Philadelphia, Pa., pp. 581-618, 1975.

[12] J. C. Hudson and K. C. Kapur, "Modules in Coherent Multistate Systems," IEEE Transactions on Reliability, vol. R-32, no. 2, pp. 183-185, 1983.

[13] Z. Birnbaum, On the Importance of Different Components in a Multi-Component System, Academic Press, New York, NY, USA, 1969.

[14] D. A. Butler, "A complete importance ranking for components of binary coherent systems, with extensions to multistate systems," Naval Research Logistics Quarterly, vol. 26, no. 4, pp. 565-578, 1979.

[15] W. S. Griffith, "Multistate reliability models," Journal of Applied Probability, vol. 17, no. 3, pp. 735-744, 1980.

[16] E. Zaitseva and V. Levashenko, "Multiple-valued logic mathematical approaches for multi-state system reliability analysis," Journal of Applied Logic, vol. 11, no. 3, pp. 350-362, 2013.

[17] S. M. Wu, "Joint importance of multistate systems," Computers and Industrial Engineering, vol. 49, no. 1, pp. 63-75, 2005.

[18] P. Wei, Z. Lu, and J. Song, "Variable importance analysis: A comprehensive review," Reliability Engineering and System Safety, vol. 142, pp. 399-432, 2015.

[19] F. Cadini, F. Santos, and E. Zio, "An improved adaptive krigingbased importance technique for sampling multiple failure regions of low probability," Reliability Engineering \& System Safety, vol. 131, pp. 109-117, 2014.

[20] D. Zhang, Y. Zhang, M. Yu, and Y. Chen, "Reliability evaluation and component importance measure for manufacturing systems based on failure losses," Journal of Intelligent Manufacturing, vol. 28, no. 8, pp. 1859-1869, 2017.

[21] D. Zhang, Y. Zhang, M. Yu, and Y. Chen, "Reliability Defects Identification of Serial Production Systems: Application to a Piston Production Line," Arabian Journal for Science and Engineering, vol. 39, no. 12, pp. 9113-9125, 2014.

[22] H. Dui, S. Si, L. Cui, Z. Cai, and S. Sun, "Component importance for multi-state system lifetimes with renewal functions," IEEE Transactions on Reliability, vol. 63, no. 1, pp. 105-117, 2014.

[23] G. Levitin, L. Podofillini, and E. Zio, "Generalised importance measures for multi-state elements based on performance level restrictions," Reliability Engineering \& System Safety, vol. 82, no. 3, pp. 287-298, 2003.

[24] M. Kvassay, E. Zaitseva, and V. Levashenko, "Importance analysis of multi-state systems based on tools of logical differential calculus," Reliability Engineering \& System Safety, vol. 165, pp. 302-316, 2017.

[25] X. Y. Zhu, M. Boushaba, D. W. Coit, and A. Benyahia, "Reliability and importance measures for m-consecutive-k, 1-out-ofn system with non-homogeneous Markov-dependent components," Reliability Engineering and System Safety, vol. 167, pp. 19, 2017.

[26] H. Dui, S. Si, and R. C. M. Yam, "Importance measures for optimal structure in linear consecutive-k-out-of-n systems," Reliability Engineering and System Safety, vol. 169, 2018.

[27] J. Bi, "A review of statistical methods for determination of relative importance of correlated predictors and identification of drivers of consumer liking," Journal of Sensory Studies, vol. 27, no. 2, pp. 87-101, 2012.

[28] W. Kuo and X. Zhu, "Importance Measures in Reliability, Risk, and Optimization: Principles and Applications," Importance Measures in Reliability, Risk, and Optimization: Principles and Applications, 2012.

[29] B. Natvig, "On the reduction in remaining system lifetime due to the failure of a specific component," Journal of Applied Probability, vol. 19, no. 3, pp. 642-652, 1982.

[30] B. Natvig and J. Gåsemyr, "New results on the Barlow-Proschan and Natvig measures of component importance in nonrepairable and repairable systems," Methodology and Computing in Applied Probability, vol. 11, no. 4, pp. 603-620, 2009.

[31] Y. Z. Zhang, J. T. Liu, G. X. Shen, X. Y. Qi, and Z. Long, "Reliability modeling of CNC machine tools system based on failure correlation analysis," Journal of Jilin University, vol. 47, no. 1, pp. 169-173, 2017. 


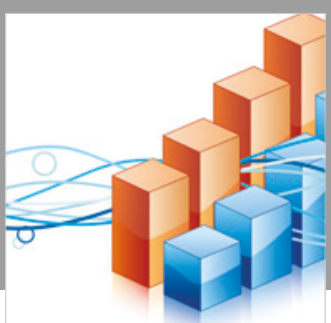

Advances in

Operations Research

\section{-n-m}
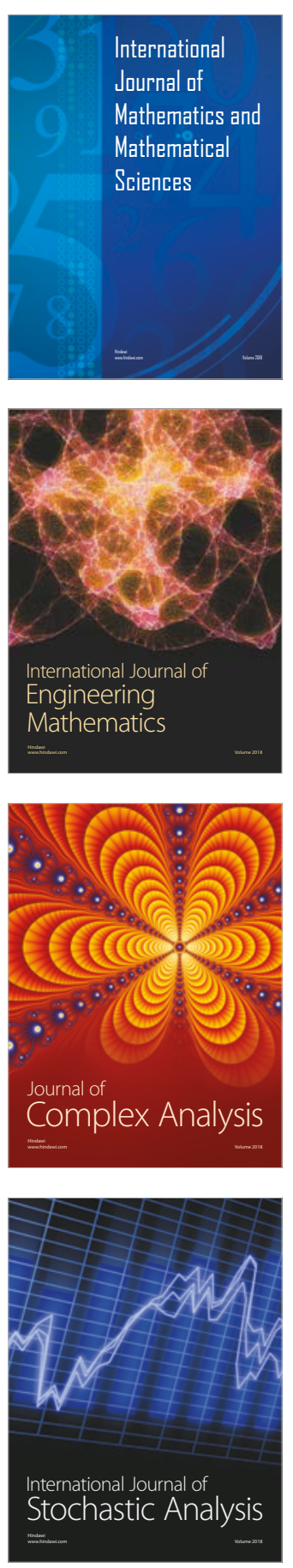
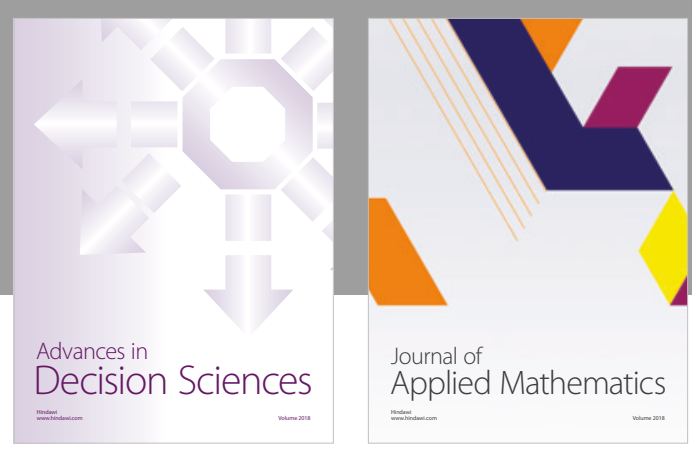

Journal of

Applied Mathematics
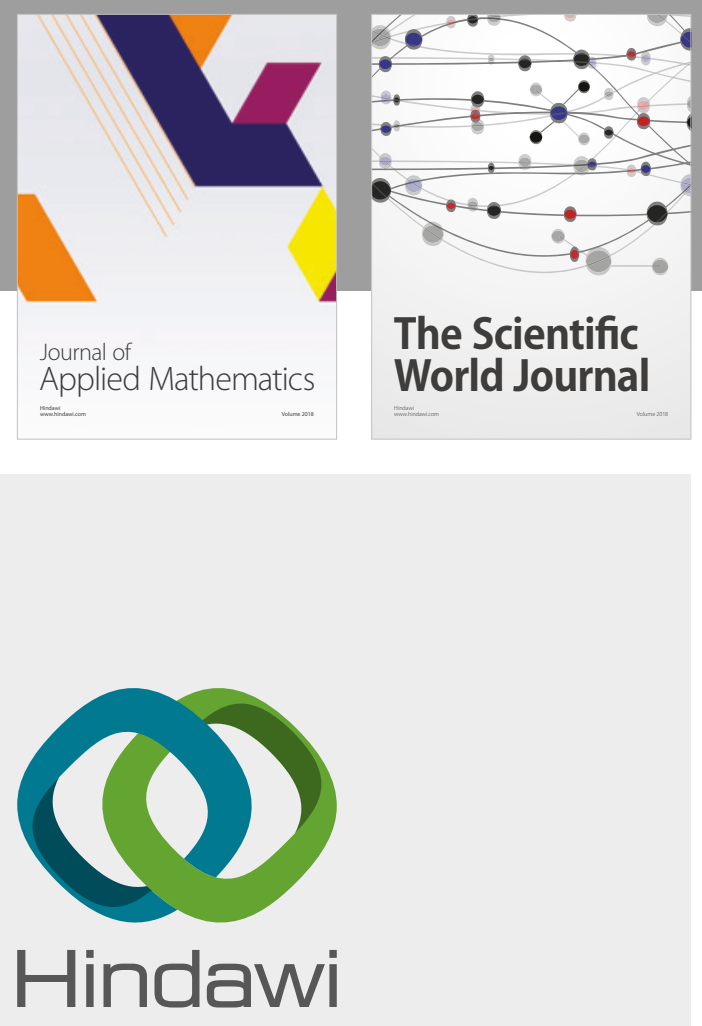

Submit your manuscripts at

www.hindawi.com

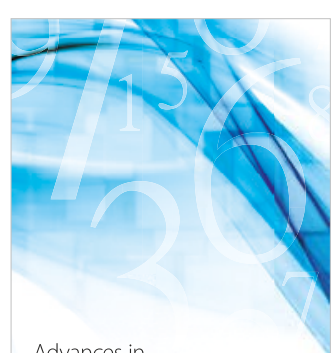

Advances in
Numerical Analysis
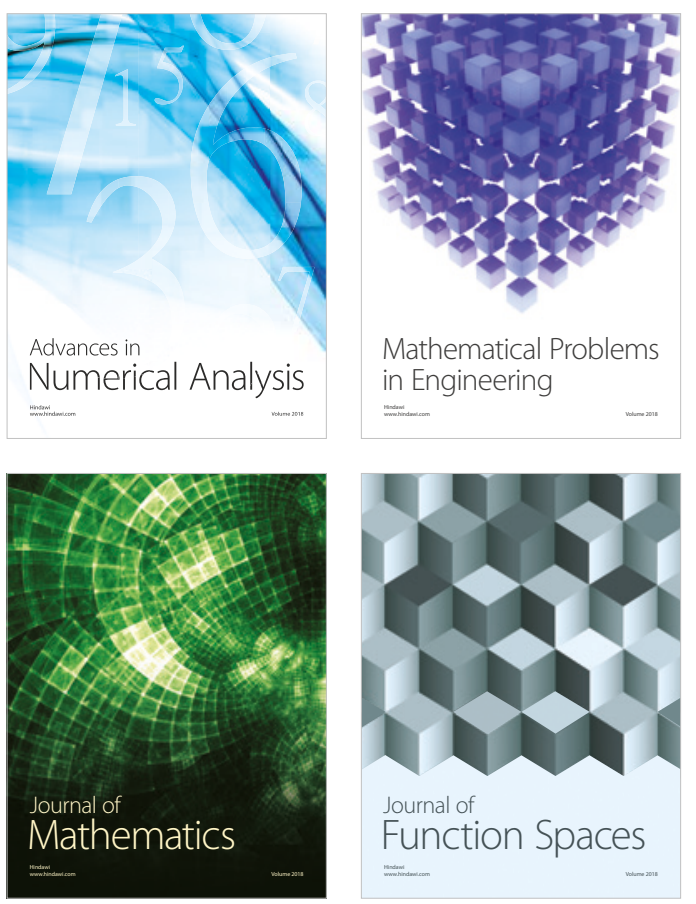

Mathematical Problems in Engineering

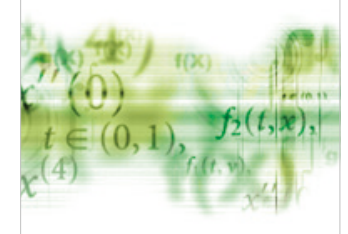

International Journal of

Differential Equations

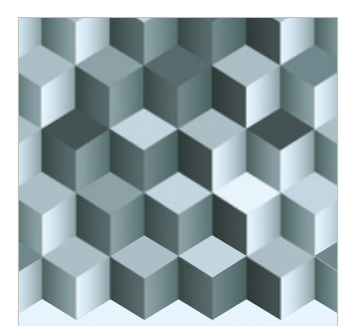

Journal of

Function Spaces

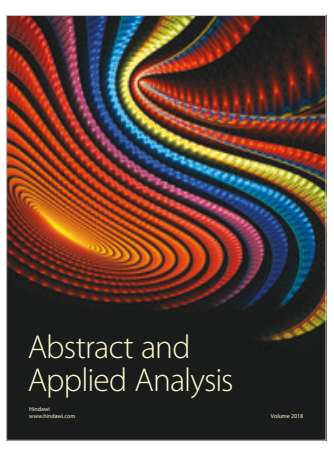

The Scientific

World Journal

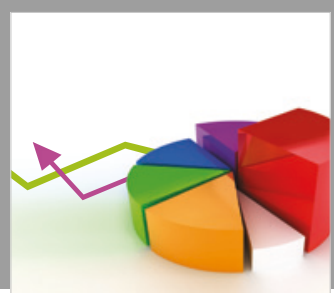

Journal of

Probability and Statistics
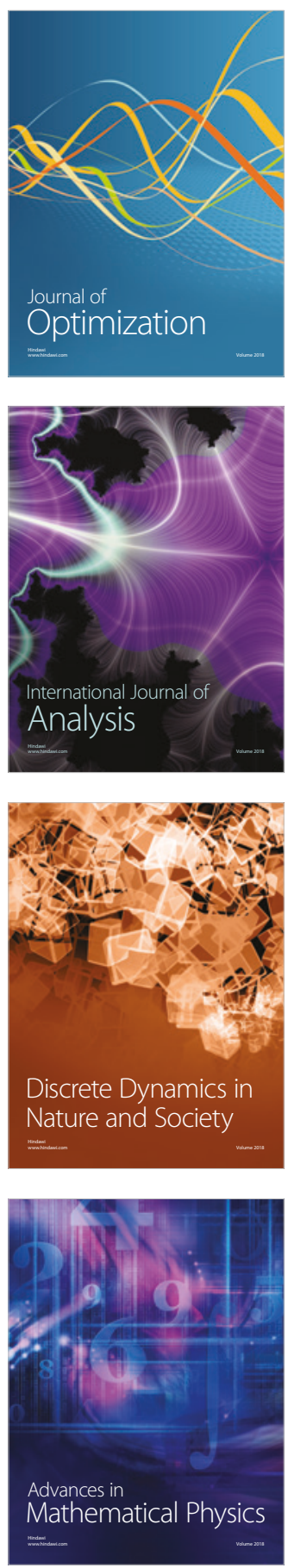